\title{
Image Reconstruction via Manifold Constrained Convolutional Sparse Coding for Image Sets
}

\author{
Linlin Yang ${ }^{1 *}, \mathrm{Ce} \mathrm{Li}^{2 *}$, Jungong $\mathrm{Han}^{3}$, Qixiang $\mathrm{Ye}^{4}$, Chen $\mathrm{Chen}^{5}$, Xianbin $\mathrm{Cao}^{6}$, Baochang Zhang ${ }^{1 \dagger}$, \\ Wanquan $\mathrm{Liu}^{7}$
}

\begin{abstract}
Convolution sparse coding (CSC) has attracted much attention recently due to its advantages in image reconstruction and enhancement. However, the coding process suffers from perturbations caused by variations of input samples, as the consistence of features from similar input samples are not well addressed in the existing literature. In this paper, we will tackle this feature consistence problem from a set of samples via a proposed manifold constrained convolutional sparse coding (MCSC) method. The core idea of MCSC is to use the intrinsic manifold (Laplacian) structure of the input data to regularize the traditional CSC such that the consistence between features extracted from input samples can be well preserved. To implement the proposed MCSC method efficiently, the alternating direction method of multipliers (ADMM) approach is employed, which can consistently integrate the underlying Laplacian constraints during the optimization process. With this regularized data structure constraint, the MCSC can achieve a much better solution which is robust to the variance of the input samples against over-complete filters. We demonstrate the capacity of MCSC by providing the state-of-the-art results when applied it to the task of reconstructing light fields. Finally, we show that the proposed MCSC is a generic approach as it also achieves better results than the state-of-the-art approaches based on convolutional sparse coding in other image reconstruction tasks, such as face reconstruction, digit reconstruction and image restoration.
\end{abstract}

Index Terms—Light Field, Image Reconstruction, Image Deblurring, Convolutional Sparse Coding, Manifold Constrained Convolutional Sparse Coding.

\section{INTRODUCTION}

W ITH the increasing number of images and videos produced in our everyday life, batch data processing techniques which can deal with high-volume data containing significant illumination variation, blurring, and severe noise, are highly demanded. For instance, high-resolution light field imaging is a typical batch system applied on a set of images captured from large camera arrays or a handheld camera moved over time around the scene [1], [2]. To handle the challenging problem caused by high sampling rate and huge-volume samples captured in various of external conditions (i.e., lighting variations), different kinds of techniques that can reconstruct dense light fields from fewer samples are developed based on various prior assumptions made about the data [2]. When processing batch images, researchers reach a consensus that the data structure as a common prior should be given much attention, which has been evidenced first in visualizing high-dimensional data in the low-dimension space [3], [4], and then some successful applications in the learning paradigm [5], [6], [7]. Especially, the data structure embedded into a constraint model could be used to simplify the learning process while improving

- Linlin Yang and Ce Li have equal contribution to the paper.

- 1. Department of Automation, Beihang University, Beijing, China. E-mail: bczhang@buaa.edu.cn; correspondence.

- 2. China University of Mining \& Technology, Beijing, China.

- 3. School of Computing \& Communications, Lancaster University, LA1 $4 Y W, U K$.

- 4. University of Chinese Academy of Sciences, Beijing, China.

- 5. University of Central Florida, Orlando, FL, USA.

- 6. School of Electronic and Information Engineering, Beihang University

- 7. Curtin University, Perth, Australia.

Manuscript received December 30, 2016.

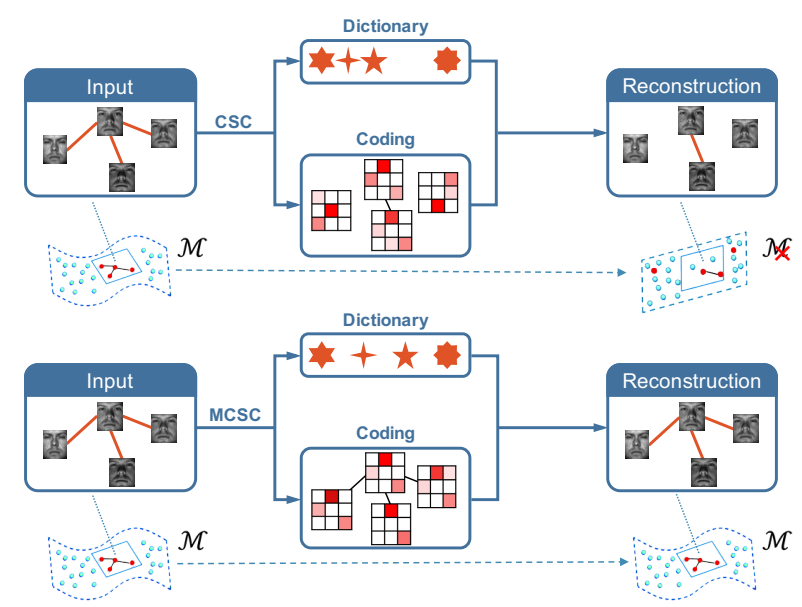

Fig. 1. Illustration of CSC and MCSC for image reconstruction. Reconstructed images by CSC that lacks structure constraint suffer from the perturbation caused by the noised or corrupted input samples. In contrast, the reconstructed images by MCSC with manifold constraint is more consistent with the input data structure. As a property of MCSC, the manifold structure of input data is preserved, leading to more reasonable reconstruction results.

the solution quality by the regularization technique, e.g., manifold regularization [5], which can be found in the latest learning algorithms [6], [7]. As detailed in [7], the constraints derived from the manifold structure in the input data can be used to improve the performance of convolution neural network (CNN). This is more important when one has to gain the robustness to variations of the input data. To this end, the methods better taking advantage of the implicit data-set structure can obtain higher performance. 
In this paper, we revisit the convolution sparse coding (CSC) [8] by considering the data on a Laplacian manifold. The CSC or deconvolution, as one of fundamental tools in machine learning, has been demonstrated to have important applications in a broad range of computer vision problems including feature learning, particularly related to the pretraining of the CNN filters [9], image deionising and/or reconstruction [9], [10], [11], [12], super-resolution [13], trajectory reconstruction [14], automatic music transcription [15] and neuronal ensemble identification [16]. The conventional CSC method was improved by new coding or dictionary learning approaches in theory [17], [18], [19]. Based on the convolution operator and sparse coding, the CSC reconstructs the vectorized input images with vectorized sparse feature maps and convolutional kernels. There are some works addressing the deficiency of the CSC based on an alternating direction method of multipliers (ADMM) approach with the mixing matrix [8]. In [20], the extended CSC has been proposed by considering local features dependency for image reconstruction as a potential direction of CSC, but the authors there only sketched out the idea with neither implementation details nor sufficient experimental results. Nevertheless, the data structure of input samples is not fully utilized in the existing CSC methods and the consistence of the features for similar input samples cannot be guaranteed. In other words, the coding results for similar input samples may vary dramatically because the neighborhood information in the data-set is ignored. With the overcomplete filter set, the coding process without consistence constraints would suffer from the perturbations caused by the variations of input samples as they might be distorted or corrupted. As shown in Fig. 1, the neighborhood of input images in the reconstruction task is emphasized via a manifold structure constraint.

In this paper, we will consider the mutual dependence among observations, which would result in large variations in the reconstructed data once it is ignored and then this provides some new insights on the CSC with manifold constraints. In particular we have an intuition on the dataset structure that was neglected: the data lying on a specific manifold can be used to improve solutions for some applications. For such purpose, we propose a new scheme of the CSC as shown in Fig. 1 for image reconstruction. Being different from the traditional CSC without structure constraint, the coding process of the proposed manifold constrained convolutional sparse coding (MCSC) is imposed to be consistent with the data structure, while the learning algorithm is implemented with the regularization technique to solve the ill-posed problem. As an important property of the MCSC, the manifold structure can be preserved, whilst leading to a better reconstruction than the conventional CSC. Furthermore, based on recent advantages in ADMM, we can efficiently solve the proposed MCSC problem based on a new manifold constraint model. One should notice that there exist some previous works to incorporate the manifold structure into deep learning [7] or sparse coding [6], [21]. However, to the best of our knowledge, this is the first time that manifold constraint is introduced for the CSC. In summary, we have the following contributions in this paper.

1. The manifold structure considered as a constraint is embedded into the CSC and the proposed problem is theoretically investigated, by which the consistence of features for similar input samples can be preserved. Consequently, the reconstruction performance is improved as the implicit manifold structure is preserved.

2. The derived theoretical results show that imposing Laplacian manifold constraint on the input data is equivalent to imposing regularization terms on the coding and dictionary, which provides an insight for other similar methods [6], [21].

3. Extensive experimental results on image reconstruction, image de-blurring and light field reconstruction show a consistent improvement of performance with respect to the existing state-of-the-art algorithms based on CSC.

The rest of the paper is organized as follows. Section 2 introduces the related works, and Section 3 describes the details of the proposed method. Experiments and results are presented in Section 4, and Section 5 concludes the paper.

\section{Related Work}

Different from existing sparse coding algorithms for classification, the reconstruction and coding tasks that assume independent observations during the learning procedure, the conventional CSC explicitly models local interactions through the convolution operator, and the resulting optimization is considerably more complicated than traditional sparse coding, especially in light field reconstruction.

In [9], [10], [11], [12], some hierarchical models that learn image decompositions via the CSC are proposed in feature learning. Moreover, an efficient feed-forward encoder that can predict sparse features was proposed for classification, which is particularly used to train the CNN filters [9], [22]. With the guarantee that neural activity is sparse in both space and time, Andilla et al. [16] proposed a sparse deconvolution approach in both space and time domain for calcium image sequences. Serrano et al. [23] verified the CSC on the high-speed video analysis task. The CSC also has attracted growing attention in processing different reconstruction tasks. Wohlberg et al. [17] proposed an extended CSC algorithm by using an augmented dictionary to incorporate the impulse noise images. Heide et al. [8] proposed a splitting-based ADMM approach with the mixing matrix to process the incomplete images and Serrano et al. [18] proposed a variant of CSC to recover high dynamic range images (HDRI), which achieved superior performances. In addition to the applications mentioned above, the CSC also has been utilized in super-resolution [13], trajectory reconstruction [14], automatic music transcription [15] and neuronal ensemble identification [16]. Meanwhile, based on recent advances in optimization [24], [25], varieties of efficient approaches to solve the CSC problems have been proposed, such as, the frequency domain method [26] and the splitting-based ADMM approach [8].

Recently, researchers have started to explore compressive light field acquisition with a single camera. The optical coding strategies include coded apertures [27], [28], coded lenslets [27], and a combination of coded mask and aperture [29].The conventional methods require multiple images to be recorded and are not suitable for reconstructing dynamic scenes, even though they succeed in reducing the number 
of shots compared to their non-compressive counterparts. Marwah et al. [1] used sparse coding techniques to learn a dictionary of basis vectors for representing light fields, in which the dictionary is chosen so that training light fields may be represented as sparse vectors. The underlying assumption is that new light fields will have similar structure to those in their training data. Similarly, Shi et al. [2] proposed the continuous sparse structure in the Fourier domain for light field reconstruction.

Different from the existing works, we present here a new method that exploits Laplacian structure as constraints in the ADMM framework, in which we emphasize on the dependence among samples into the CSC. As a theoretical contribution, we show that the Laplacian manifold structure from data samples can be considered as regularization terms imposed on coding coefficients and dictionary. This makes our work significantly different from [21], where the Laplacian regularization on coding coefficients is used only for sparse coding. Instead, we focus on the convolution sparse coding with Laplacian regularization on both coding and dictionary learning in the ADMM framework. Our method is also different from [20], which is based on an assumption that Laplacian regularization is imposed on only a single image, whereas we focus on a batch of images.

\section{Manifold Constrained CSC}

In this section, we present how manifold can be incorporated into the CSC as a constraint and then construct the MCSC. We then solve the MCSC problem in the ADMM framework with theoretical analysis.

\subsection{Laplacian Manifold for the CSC}

As one of increasingly important tools in machine learning and computer vision, the CSC method can be used to learn the features subsequently used for classification and reconstruction tasks. Based on the convolution operator and sparse coding, the CSC is formulated as:

$$
\begin{array}{ll}
\underset{\mathbf{d}, \mathbf{z}}{\operatorname{minimize}} & \frac{1}{2} \sum_{n=1}^{N}\left\|\mathbf{y}^{n}-\sum_{k=1}^{K} \mathbf{d}_{k} * \mathbf{z}_{k}^{n}\right\|_{2}^{2}+\beta \sum_{n=1}^{N} \sum_{k=1}^{K}\left\|\mathbf{z}_{k}^{n}\right\|_{1} \\
\text { subject to } & \left\|\mathbf{d}_{k}\right\|_{2}^{2} \leq 1 \quad \forall k \in\{1, \ldots, K\},
\end{array}
$$

where $\mathbf{y}^{n} \in R^{L_{1}}$ are vectorized input images, $\mathbf{z}_{k}^{n} \in R^{L_{1}}$ are vectorized sparse feature maps and $\mathbf{d}_{k} \in R^{L_{2}}$ are the vectorized convolutional kernels. $N$ is the number of input images, $\beta$ is the sparsity parameter, $K$ is the number of the kernels and $*$ is the $2 \mathrm{D}$ convolution operator defined on the vectorized inputs. The formulation (P1) can be generally customized for different applications. In the above formulation, it is assumed that $\mathbf{y}^{n} \in R^{L_{1}}$ are independent. We assume that the input samples $\mathbf{y}^{n} \in R^{L_{1}}$ are lying on a manifold. To utilize the data structure prior, we first reformulate $(\mathrm{P} 1)$ as:

$$
\begin{array}{cl}
\underset{\mathbf{d}, \mathbf{z}}{\operatorname{minimize}} & \frac{1}{2}\|\mathbf{y}-\mathbf{H v}\|_{F}^{2}+\beta \sum_{n=1}^{N} \sum_{k=1}^{K}\left(\left\|\mathbf{z}_{k}^{n}\right\|_{1}+\frac{1}{2}\left\|\mathbf{z}_{k}^{n}\right\|_{2}^{2}\right) \\
\text { subject to } & \left\|\mathbf{d}_{k}\right\|_{2}^{2} \leq 1 \quad \forall k \in\{1, \ldots, K\} \\
& \mathbf{v}^{\mathbf{n}} \in \mathcal{M},
\end{array}
$$

where $\mathbf{v}^{n}=\sum_{k=1}^{K} \mathbf{d}_{k} * \mathbf{z}_{k}^{n}, \mathbf{v}=\left[\mathbf{v}^{1}, \ldots, \mathbf{v}^{N}\right], \mathbf{y}=$ $\left[\mathbf{y}^{1}, \ldots, \mathbf{y}^{N}\right] . \quad \mathcal{M}$ is a set of samples representing the underlying manifold. To encourage a grouping effect, where strongly correlated filters tend to be in or out of the model together, we choose elastic net constraints [30] instead of sparsity constraints. $\mathbf{H}$ is a diagonal matrix whose diagonal entries are either 1 or 0 , keeping or killing the corresponding pixels for image reconstruction, or a measurement matrix containing the sheared mask code of multiple recorded sensors for light filed, or a blur matrix given by point spread function (PSF) and boundary condition for de-blurring, which allows us to gain the robustness in the CSC framework for the complex situations, such as motion blur, outof-focus blur and illumination variation. As a basic intuition, the manifold structure contained in the data set $\mathbf{y}^{n} \in R^{L_{1}}$ should be remained in the convolutional coding process. In particular $\mathbf{y}^{n} \in \mathcal{M}$ are the observations of degraded images, and $\mathbf{v}^{n} \in \mathcal{M}$ are the reconstructions of $\mathbf{y}^{n}$ that are thus subject to a manifold constraint. Again we expect the dependence among samples would help us obtain better coding results as well as better solution for the filter learning.

It should be noted that the manifold structure in the original input data is usually not accurate due to the fact that the input data may suffer from noise or corruption. In comparison, we expect the manifold structure becomes more accurate based on $\mathbf{v}$ as a better reconstruction after removing noise or corruption. Moreover, we prove that the manifold constraints in terms of $\mathbf{v}$ can be interpreted to be the meaningful regularizations in ADMM, which can improve the kernels' orthogonally and features' neighbor relationship. The proof details are presented in Section 3.3.

In order to solve (P2) with structure preservation, $\mathbf{v}^{n} \in$ $\mathcal{M}$ is the only unsolved part. We use the manifold in a constrained model that is different from the most popular approaches such as the Local Linear Embedding (LLE) [3] and the Laplacian with a focus on the data structure visualization. In contrast, the Laplacian Eigenmap [4] provides a computationally efficient approach to nonlinear dimensionality reduction that has locality-preserving properties with a natural connection to clustering. Here, we calculate the Laplacian structure of $\mathbf{y}$ in the input space that can be transferred to $\mathbf{v}$ via a specific constraint function. Technically, the Laplacian matrix $\mathbf{L}$ [4] is used to replace $\mathbf{v}^{n} \in \mathcal{M}$ in P2 and thus we can obtain the following MCSC formulation.

$$
\begin{array}{cl}
\underset{\mathbf{d}, \mathbf{z}}{\operatorname{minimize}} & \frac{1}{2}\|\mathbf{y}-\mathbf{H v}\|_{F}^{2}+\frac{\gamma}{2} \operatorname{tr}\left(\mathbf{v} \mathbf{L} \mathbf{v}^{T}\right) \\
& +\beta \sum_{n=1}^{N} \sum_{k=1}^{K}\left(\left\|\mathbf{z}_{k}^{n}\right\|_{1}+\frac{1}{2}\left\|\mathbf{z}_{k}^{n}\right\|_{2}^{2}\right) \\
\text { subject to } & \left\|\mathbf{d}_{k}\right\|_{2}^{2} \leq 1 \quad \forall k \in\{1, \ldots, K\},
\end{array}
$$

where $\mathbf{L}$ is a Laplacian matrix with a parameter $\gamma$, which weights the contribution of the regularization term. The solution to Eq. 1 with a new manifold constraint function $\frac{\gamma}{2} \operatorname{tr}\left(\mathbf{v L} \mathbf{v}^{T}\right)$ will be investigated based on the ADMM framework in the next subsection. It is actually the only part where manifold plays a role in (P2). According to [5], the nonlinear structure as a resulting regularization term can avoid over-fitting and ensure that a problem is well-posed by penalizing complicated solutions. 


\subsection{Solution to (P2) Based on the ADMM}

The main optimization tool we use in solving this convex problem Eq. 1 is ADMM, which is a variant of the standard Augmented Lagrangian Multiplier (ALM) method that uses partial updates (similar to the Gauss-Seidel method for solving linear equations). The ADMM recently has received much more attention because of its adaptability to several problems [24]. By iteratively solving a set of simple convex optimization sub-problems, one can obtain the final solution of (P2). More details about ADMM can be found in [8], [24], [25], [31]. The ADMM can solve the sub-convex problems for two or more variables when one treats one variable each time while other variables are fixed. By exploiting the merits of the ADMM, we develop three update steps as described below: coding update, dictionary update and structure update. The coding update aims for more precise reconstruction coefficients, dictionary update for better representation and structure update for data manifold structure constraint.

First, the coding update is achieved by:

$$
\begin{array}{ll}
\underset{\mathbf{z}}{\operatorname{minimize}} & \frac{1}{2}\|\mathbf{y}-\mathbf{H} \mathbf{v}\|_{F}^{2}+\frac{\gamma}{2} \operatorname{tr}\left(\mathbf{v} \mathbf{L} \mathbf{v}^{T}\right) \\
& +\beta \sum_{n=1}^{N} \sum_{k=1}^{K}\left(\left\|\mathbf{z}_{k}^{n}\right\|_{1}+\frac{1}{2}\left\|\mathbf{z}_{k}^{n}\right\|_{2}^{2}\right),
\end{array}
$$

where $\mathbf{v}^{n}=\mathbf{D} \mathbf{z}^{n}, \mathbf{D}=\left[\mathbf{D}_{1}, \ldots, \mathbf{D}_{K}\right]$ is a concatenation of Toeplitz matrix representing a convolution with the respective filter $\mathbf{d}_{k}, \mathbf{z}=\left[\mathbf{z}^{1}, \ldots, \mathbf{z}^{N}\right], \mathbf{z}^{n}=\left[\mathbf{z}_{1}^{n T}, \ldots, \mathbf{z}_{k}^{n T}\right]^{T}$.

Next, the dictionary update problem is defined as:

$$
\begin{array}{ll}
\underset{\mathbf{d}}{\operatorname{minimize}} & \frac{1}{2}\|\mathbf{y}-\mathbf{H v}\|_{F}^{2}+\frac{\gamma}{2} \operatorname{tr}\left(\mathbf{v} \mathbf{L} \mathbf{v}^{T}\right) \\
\text { subject to } & \left\|\mathbf{d}_{k}\right\|_{2}^{2} \leq 1 \quad \forall k \in\{1, \ldots, K\},
\end{array}
$$

where $\mathbf{v}^{n}=\mathbf{Z}^{n} \mathbf{d}, \mathbf{Z}^{n}$ is a concatenation of Toeplitz matrices for the respective sparse codes $\mathbf{z}_{k}^{n}, \mathbf{Z}=\left[\mathbf{Z}_{1}, \ldots, \mathbf{Z}_{N}\right], \mathbf{d}=$ $\left[\mathbf{d}_{1}^{T}, \ldots, \mathbf{d}_{K}^{T}\right]^{T}$.

And finally, the structure update is shown as:

$$
\mathbf{L}=(1-\eta) \mathbf{L}+\eta L E(\mathbf{D z}),
$$

where $\mathbf{L}$ is initialized equal to $L E(\mathbf{y})$, and $L E(\cdot)$ means normalized Laplacian matrix function defined in [4] and $\eta$ represents update ratio.

To simplify our ADMM, we define four functions that are used to reformulate three mentioned update steps:

$$
\begin{array}{ll}
f_{1}(\mathbf{x})=\frac{1}{2}\|\mathbf{y}-\mathbf{H} \mathbf{x}\|_{2}^{2}, & f_{2}(\mathbf{x})=\frac{\gamma}{2} \operatorname{tr}\left(\mathbf{x} \mathbf{L} \mathbf{x}^{T}\right), \\
f_{3}(\mathbf{x})=\beta\left(\|\mathbf{x}\|_{1}+\frac{1}{2}\|\mathbf{x}\|_{2}^{2}\right), & f_{4}(\mathbf{x})=i n d_{c}(\mathbf{x}),
\end{array}
$$

where $\operatorname{ind}_{c}(\cdot)$ represents the indicator function and $C=$ $\left\{\mathbf{x} \mid\|\mathbf{S x}\|_{2}^{2} \leq 1\right\}$. The proximal operators [25] used to approximate the above four objectives can be derived as

$$
\begin{aligned}
& \operatorname{prox}_{\lambda f_{1}}(\mathbf{x})=\left(\mathbf{I}+\lambda \mathbf{H}^{\mathbf{T}} \mathbf{H}\right)^{-1}\left(\mathbf{x}+\lambda \mathbf{H}^{\mathbf{T}} \mathbf{y}\right) \\
& \operatorname{prox}_{\lambda f_{2}}(\mathbf{x})=\mathbf{x}(\gamma \lambda \mathbf{L}+\mathbf{I})^{-1} \\
& \operatorname{prox}_{\lambda f_{3}}(\mathbf{x})=\left(\frac{1}{1+\lambda}\right)\left(\max \left(1-\frac{\lambda}{|\mathbf{x}|}, 0\right) \odot \mathbf{x}\right) \\
& \operatorname{prox}_{\lambda f_{4}}(\mathbf{x})=\left\{\begin{array}{cl}
\frac{\mathbf{S} \mathbf{x}}{\|\mathbf{S x}\|_{2}^{2}} & \|\mathbf{S x}\|_{2}^{2} \geq 1 . \\
\mathbf{S x} & \text { else }
\end{array}\right.
\end{aligned}
$$

In Alg. 1, we show the iterative steps of the MCSC which can solve P2 efficiently.

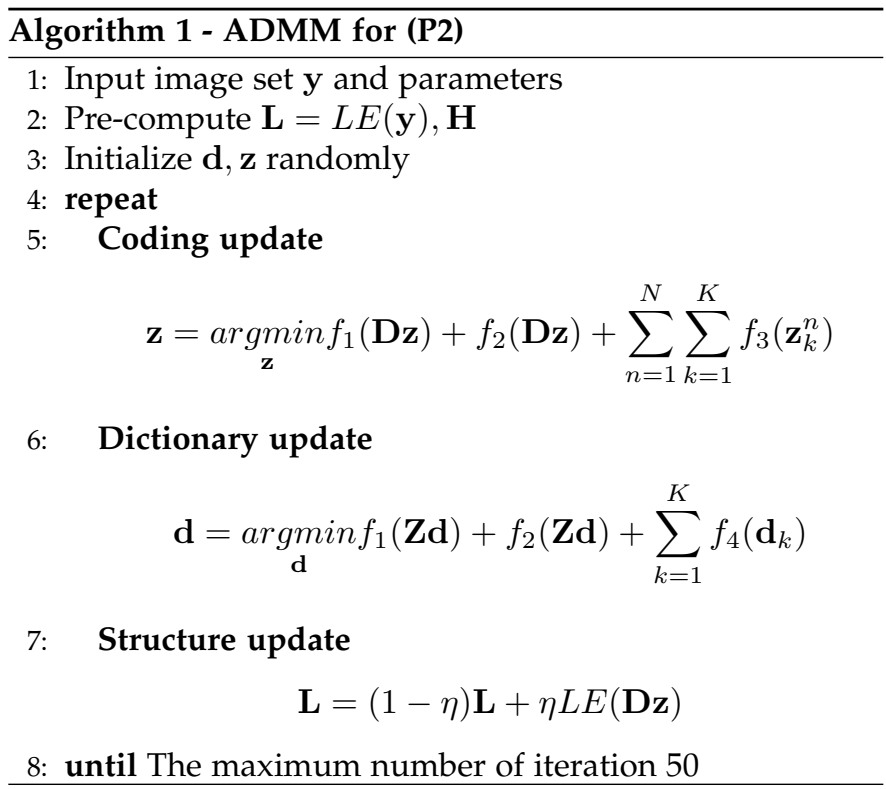

\subsection{Theoretic Analysis of the MCSC}

In this subsection, we conduct theoretical analysis for the proposed MCSC in two theorems. In detail, Theorem 1 states that the Laplacian structure in input data $\mathbf{v}$ can approximately constrain the solution as that in feature space $\mathbf{z}$ ( $\mathrm{min}$ imizing $\operatorname{tr}\left(\mathbf{v} L \mathbf{v}^{\mathbf{T}}\right)$ is approximated as $\left.\operatorname{tr}\left(\mathbf{z} L \mathbf{z}^{\mathbf{T}}\right)\right)$. Theorem 2 proves that in the dictionary update step the Laplacian structure constraint in input data is in fact equal to the constraint imposed on the dictionary (l2-norm). We describe two lemmas [32] and then prove our main theorems. The proofs of lemmas and theorems are shown in the Appendix.

Theorem 1: Given D, and a positive constant $\alpha^{\prime}$, we have:

$$
\begin{aligned}
c_{1} \operatorname{tr}\left(\mathbf{z} \mathbf{L} \mathbf{z}^{T}\right) & \leq \operatorname{tr}\left(\mathbf{v} \mathbf{L} \mathbf{v}^{T}\right)+\alpha^{\prime} \sum_{n=1}^{N} \sum_{k=1}^{K}\left\|\mathbf{z}_{k}^{n}\right\|_{2}^{2} \\
& \leq c_{2} \operatorname{tr}\left(\mathbf{z L} \mathbf{z}^{T}\right)+\alpha^{\prime} \sum_{n=1}^{N} \sum_{k=1}^{K}\left\|\mathbf{z}_{k}^{n}\right\|_{2}^{2},
\end{aligned}
$$

where $c_{1}$ and $c_{2}$ are both positive constants.

We observe in this theorem that as a property of the MCSC, minimizing $\operatorname{tr}\left(\mathbf{v} \mathbf{L v} \mathbf{v}^{T}\right)$ is approximated by minimizing $\operatorname{tr}\left(\mathbf{z} \mathbf{L} \mathbf{z}^{T}\right)$. In other words the constraint on the data $\mathbf{v}$ can be transferred to the feature space $\mathbf{z}$. As mentioned above the Laplacian structure in the input data can constrain the solution as that in the feature space.

Theorem 2: Given $\mathbf{Z}$, and two positive constants $\alpha^{\prime}, \beta^{\prime}$, we have:

$$
c_{3}\|\mathbf{d}\|_{2}^{2} \leq \operatorname{tr}\left(\mathbf{v} \mathbf{L} \mathbf{v}^{T}\right)+\left(\alpha^{\prime}+\beta^{\prime}\right)\|\mathbf{d}\|_{2}^{2} \leq c_{4}\|\mathbf{d}\|_{2}^{2},
$$

where $c_{3}$ and $c_{4}$ are both positive constants. Similar to the observation in Theorem 1, minimizing $\operatorname{tr}\left(\mathbf{v} \mathbf{L} \mathbf{v}^{T}\right)$ is approximated by minimizing $l 2$-norm of $\mathbf{d}$. Again the constraint is transferred from one variable to another.

In summary, two theorems show that the Laplacian manifold structure of input data can be transferred as the 
regularization terms on optimized variables, coding coefficients or dictionary. Comparatively the nonlinear structure constraints in previous works are added on coding coefficients in a brute-force way [6], [21]. As another obvious advantage of MCSC, the manifold constraint on the data can be more explicitly represented by the optimized variables.

\section{EXPERIMENTS}

In this section, we use publicly available datasets to validate the effectiveness of the proposed approach on the reconstruction or restoration problems. The experiments are conducted on face (Yale B) [33], digits (USPS) [34], a realapplication video for car [35], and the Chess [36]. The Yale B dataset consists of 2,414 frontal-face images from 38 individuals (about 64 images per subject) captured under various laboratory-controlled lighting conditions. The USPS dataset is an image dataset including 7291 training images and 2007 test images. The video for car consists of 900 $97 \times 79$-sized images. The Chess [36] database consists of a $17 \times 17$ grid of viewpoints with $1400 \times 800$ image resolution, taken from the Stanford light field archive. The comparative methods are some state-of-the-art methods including patch based sparse coding (PSC) [1], [37], CSC [8], GSR [38] and MCSCf (see definition in section 4.2). Following the experiments in [8], [37], one part of data is used for training the dictionary and the other is for testing in reconstruction tasks. To make manifold structure of the data more transparent, we first preprocessed the input images as follows:

Scheme 1. Translating one and two pixels in each of the four directions, and rotating the images by one to three degrees.

Scheme 2. Random subsampling of the data.

Scheme 3. Adding motion blur with 0 mean and 0.01 deviations.

To evaluate the quality of the reconstructed images, the signal-to-noise ratio (PSNR, unit: $\mathrm{dB}$ ) is calculated. The higher PSNR is, the better visual quality is achieved for the reconstructed image. In our experiments, one part of data is used for training the dictionary and the other data is for testing in the reconstruction task. Three parameters are involved in the MCSC: the sparsity of coding $\beta$, the weight of manifold constraint $\gamma$ and the manifold structure update ratio $\eta$. It should be noted that the parameters are set according to different applications which might be very different, aiming to deliver a good tradeoff between sparsity and data fit. Regarding to the computational cost, the MCSC can be solved as efficiently as that of the baseline algorithm [8]. For example, the running time for digit ' 0 ' in USPS are respectively 100 minutes and 96 minutes for the MCSC and CSC, on a PC with Intel i7 CPU and 16G RAM.

\subsection{Light Field Reconstruction}

We demonstrate an application of batch light field reconstruction on the widely used Chess database [36] for MCSC. The coded 2D projection and the reconstructed light fields are shown in Fig. 2. MCSC allows for higher-resolution light fields to be reconstructed than previously possible from a single image, where $y$ is the vectorized observed sensor image, $v$ is the vectorized light field, and $H$ is a sparse measurement matrix containing the sheared coded projection operator on its diagonal, similar to [1]. In this case, there are $17 \times 17$ angular light field views stacked in $v$, the Laplacian matrix is initialized to a suitable default value and the resolution of sensor images are resized into $150 \times 100$ in our experiment. The recorded sensor image is stacked in $y$, and the corresponding measurement matrices are coded using random masks and stacked in $H$. The interesting case of $4 \mathrm{D}$ light field reconstruction is addressed to validate the performance of MCSC, in comparison with the PSC [1] and CSC [8]. Following the baseline methods [1], [8], we train $648 \times 8$-sized filters, and then we set $\beta=0.3$ in PSC and CSC, while $\beta=0.01, \gamma=0.1$ and $\eta=0.01$ in MCSC. The results are shown in Fig. 2 and Table 1. It can be observed that MCSC achieves better reconstruction quality (i.e., left-bottom of the chess board) than that of CSC, based on a compact dictionary due to the regularized manifold structure. The image can be well covered even under complex lighting conditions, occlusion and blurring.

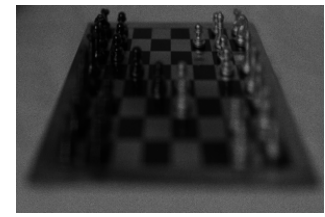

(a)

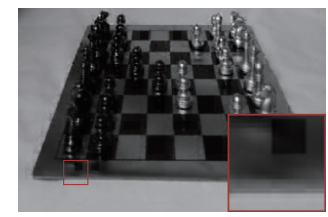

(c)

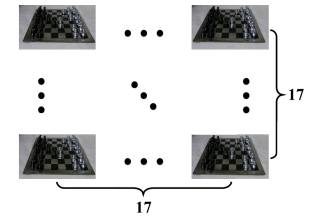

(b)

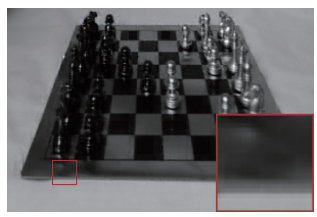

(d)
Fig. 2. (a) Coded 2D Projection; (b) Reconstructed 4D Light Field; (c) The reconstructed light field by CSC; (d) The reconstructed light field by MCSC.

TABLE 1

Average PSNR (db) for Chess and YaleB01 database.

\begin{tabular}{|c||c||c||c||c|}
\hline Method & PSC & CSC & MCSCf & MCSC \\
\hline Chess & 29.79 & 30.96 & - & $\mathbf{3 1 . 5 4}$ \\
\hline YaleB01 & 27.78 & 28.19 & 28.40 & $\mathbf{2 8 . 4 6}$ \\
\hline
\end{tabular}

\subsection{Face Reconstruction}

In this subsection, we evaluate the quality of face image reconstruction in terms of the average PSNR measure using the extended Yale Face Database B [33]. We process the images with scheme 1 . The images are first resized to $96 \times 84$ and then normalized by dividing 255 . We trained $6415 \times 15$ sized filters, and compare these MCSC filters with the PSC [37] and CSC [8]. Besides, we also compare MCSC with the MCSCf filter. Specifically, MCSCf is defined as the CSC with the manifold constraint on the feature while MCSC is the CSC with the manifold constraint on both data and feature. We set $\beta=0.3$ in the PSC and CSC, while $\beta=0.3$, $\gamma=0.1$ and $\eta=0$ in MCSCf and MCSC. Six of the reconstructions and the dictionaries are shown in Fig. 3. Fig. 3(b) and (c) show the results for a set of six faces with 


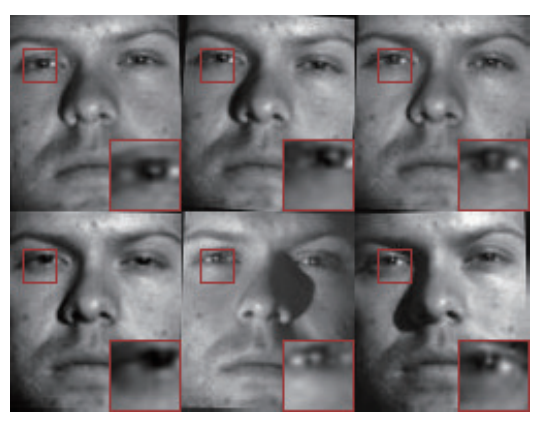

(a)

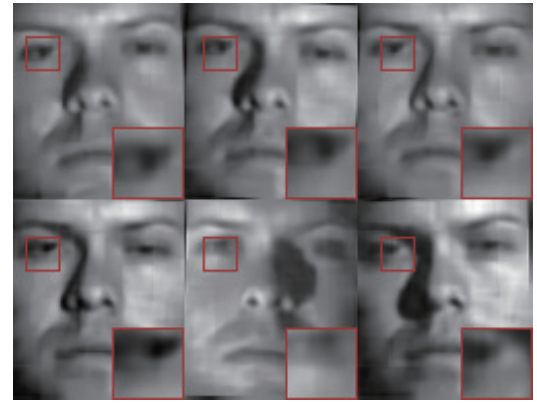

(b)

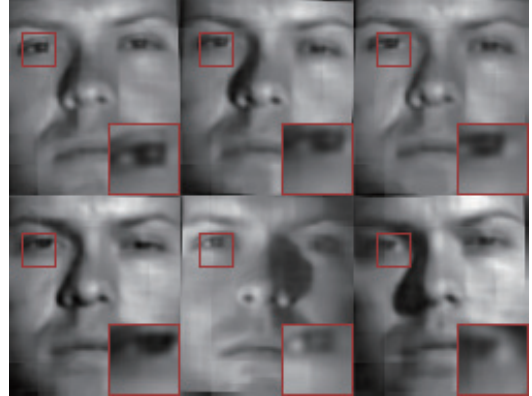

(c)

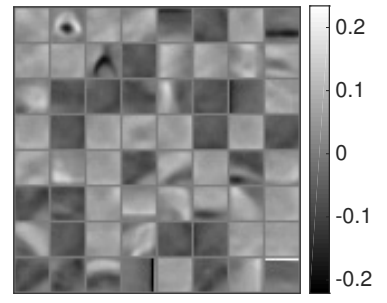

(d)

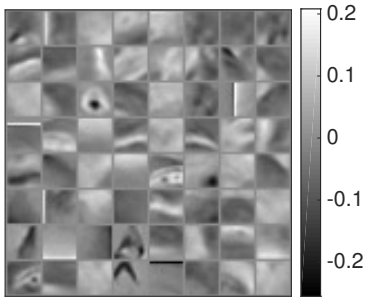

(e)

Fig. 3. (a) Original YaleB01 images; (b) Reconstruction by CSC; (c) Reconstruction by MCSC; (d) CSC dictionary; (e) MCSC dictionary.

TABLE 2

Average PSNR for different digit datasets with $257 \times 7$-sized filters.

\begin{tabular}{|c||c||c||c||c||c|}
\hline Digit & 0 & 1 & 2 & 3 & 4 \\
\hline PSC & 21.00 & 24.58 & 23.30 & 22.83 & 24.68 \\
CSC & 22.65 & 27.33 & 24.54 & 24.66 & 26.04 \\
MCSCf & 23.00 & 28.10 & 24.66 & 25.15 & 26.42 \\
MCSC & $\mathbf{2 3 . 1 4}$ & $\mathbf{2 8 . 5 0}$ & $\mathbf{2 4 . 6 6}$ & $\mathbf{2 5 . 2 0}$ & $\mathbf{2 6 . 4 2}$ \\
\hline \hline Digit & 5 & 6 & 7 & 8 & 9 \\
\hline PSC & 23.17 & 23.28 & 24.29 & 23.33 & 24.27 \\
CSC & 24.56 & 24.79 & 25.59 & 24.87 & 26.08 \\
MCSCf & 24.74 & 25.06 & 26.14 & 24.99 & 26.35 \\
MCSC & $\mathbf{2 4 . 9 5}$ & $\mathbf{2 5 . 2 3}$ & $\mathbf{2 6 . 4 0}$ & $\mathbf{2 5 . 1 4}$ & $\mathbf{2 6 . 4 2}$ \\
\hline
\end{tabular}

different reconstruction methods, respectively. All left eyes (upper left) are amplified and compared in the bottom right corner. It can be observed that MCSC achieves much better visual quality (i.e., eyes) than that of CSC, because of the compactness of dictionary (Fig. 11) that contributes to better reconstruction results. In terms of the average PSNR values in Table 1, MCSCf and MCSC improve CSC by $0.21 \mathrm{~dB}$ and $0.27 \mathrm{~dB}$ respectively. It is also obvious that the MCSC achieves a higher performance than other methods.

\subsection{Digit Reconstruction}

We perform handwritten digit reconstruction on the widely used the USPS database [34]. We experimented on digit ' 0 ' to ' 9 ' respectively with locally contrast normalization [8]. We adopt the parameters in [8] and set $\beta=1$ in the PSC and CSC, while $\beta=0.8, \gamma=0.4, \eta=0$ in MCSCf and MCSC. As baselines, the results of PSC [37] and CSC [8] are also listed in Table 2. Moreover, the reconstructions for digit ' 9 ' have been shown in the amplified images in the bottom right corner of Fig. $4^{1}$. One can see that the proposed MCSC achieves a better performance than the compared methods.

1. We show 100 out of the total reconstructions and dictionaries.
To further validate the proposed method, we also conduct the experiment of digit recognition. In experiments, 200 training images per class are randomly seletected for training and 2007 test images are selected for testing. 9 dictionaries with $7 \times 7$-sized filters are trained for each class, and the parameters are set in the same way as in [37]. We repreat this procedure 10 times. The average recognition accuracy shown in Table 3 presents the preliminary evidence of the capability of MCSC for pattern recognition task.

TABLE 3

Average recognition accuracy (\%) of four methods on USPS database.

\begin{tabular}{|c||c||c||c||c|}
\hline Method & PSC & CSC & MCSCf & MCSC \\
\hline Average Accuracy & 97.11 & 97.50 & 97.95 & $\mathbf{9 8 . 0 5}$ \\
\hline
\end{tabular}

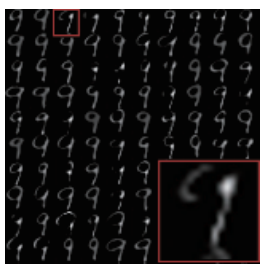

(a)

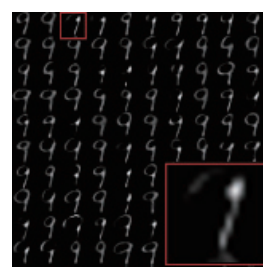

(b)

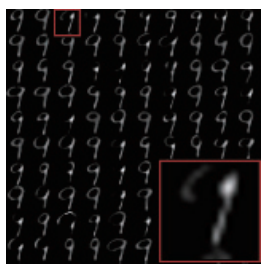

(c)

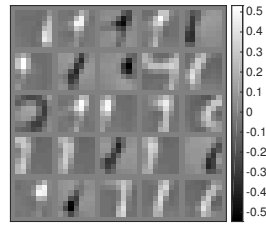

(d)

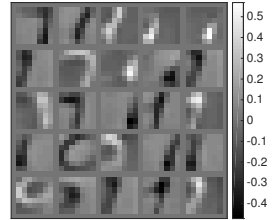

(e)
Fig. 4. (a) Original digit '9' images; (b) Reconstruction by CSC; (c) Reconstruction by MCSC; (d) CSC dictionary; (e) MCSC dictionary. 


\subsection{Image Restoration}

Batch image restoration is to reconstruct the original image from degraded observations. As a typical application of the MCSC, one can apply it to different kind of restoration problems as long as appropriate $H$ is given and also an offset term as a smoothness prior [8], [18]. Here, $H$ is a diagnoal matrix or PSF, about the selection of $H$ in our method is discussed for (P2). Two interesting cases of image restoration, i.e., inpainting and deblurring, are addressed to validate the performance of the proposed MCSC, in comparison with the CSC and group-based sparse representation (GSR) [38]. We optimally choose $\beta=0.05$ in the GSR and CSC, while $\beta=0.05, \gamma=0.01$ and $\eta=0.01$ in the MCSC. For inpainting, we process the digit ' 0 ' in USPS dataset with scheme 2. Inpainting restoration results of ' 0 ' (100 out of the total) are shown in the bottom right of Fig. 5 and Table 4. To further validate the effectiveness of the structure constraints, we test the parameters $\gamma=0.01, \gamma=0.5$, and $\gamma=2$, for image inpainting as shown in Fig. 5(d)-(f), respectively. Obviously, the parameter $\gamma$ influences the completeness of the digit ' 0 ', which again validates the effectiveness of the manifold constraint in the MCSC. For deblurring, we use the video car selected from a public dataset [35] that is further processed with scheme 3 . The deblurring restoration results ( 2 out of 900) are compared in Fig. 6 and Table 4. Again, the MCSC achieves the best reconstruction results, due to the regularized manifold structure imposed on the CSC.

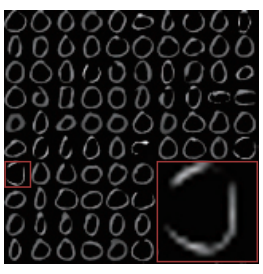

(a)

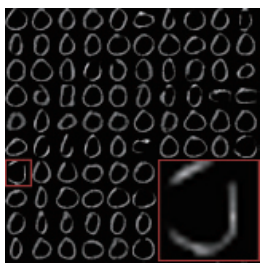

(d)

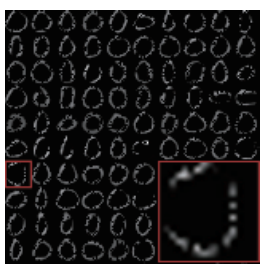

(b)

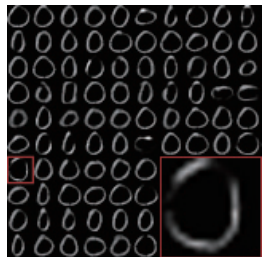

(e)

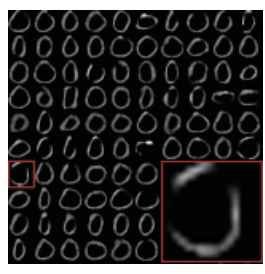

(c)

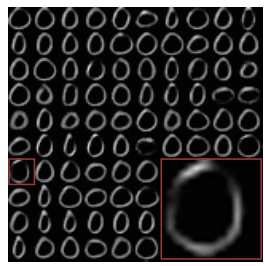

(f)
Fig. 5. Comparison of different inpainting methods for $75 \%$ subsampling (a) Original digit ' 0 ' images; (b) Subsampled images; (c) The inpainted images by CSC; (d) The inpainted images by MCSC $(\gamma=0.01, \eta=$ $0.01)$; (e) The inpainted images by MCSC $(\gamma=0.5, \eta=0.01)$; (f) The inpainted images by MCSC $(\gamma=2, \eta=0.01)$.

TABLE 4

Average PSNR of inpainting methods for different subsampling rates and deblurring methods.

\begin{tabular}{|c||c||c||c||c|}
\hline Method & Subsample & GSR & CSC & MCSC \\
\hline Inpainting for $75 \%$ & 22.31 & 34.64 & 35.16 & 35.44 \\
\hline Inpainting for $50 \%$ & 19.35 & 29.31 & 30.19 & 30.59 \\
\hline \hline Method & Motion blur & GSR & CSC & MCSC \\
\hline Deblurring & 23.37 & 33.89 & 34.13 & 34.24 \\
\hline
\end{tabular}

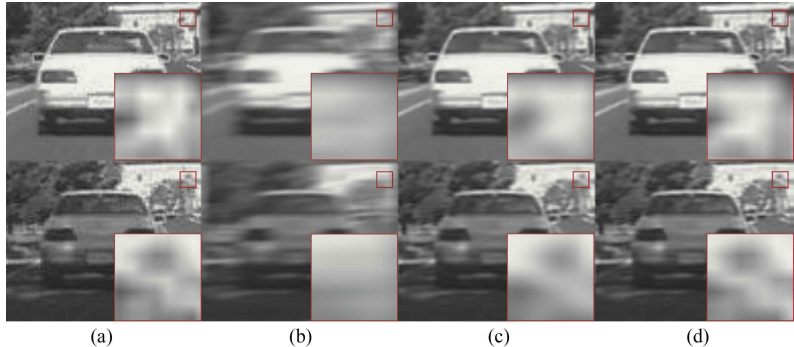

Fig. 6. Comparison of different deblurring methods. (a) Original car images; (b) Motion blur images; (c) The deblurred images by CSC; (d) The deblurred images by MCSC.

\subsection{Discussion of the MCSC}

As shown in the above experiments, MCSC achieves a higher performance than the well-known baseline CSC. This section further discusses how a higher performance has been achieved. We illustrate the performance of MCSC and CSC based on the reconstruction error, which is an important metric to evaluate for evaluating the quality of image reconstruction. A smaller value denotes less information loss. But when we process batch images, the reconstruction error can not be used to valuate the quality of data structure. Even for a same reconstruction error, manifold structures may vary a lot. Differently we focus on both reconstruction error and structure illustration. Imposing the manifold constraint is expected to better preserve the data structure and decrease the reconstruction loss.

Manifold Structure. In Fig. 7, we plot manifold structures of the input data, CSC and MCSC reconstructions on digit ' 0 ' in USPS. The dataset has more than 1000 images, based on which we estimate the structures using the Laplacian Eigenmaps [4] with the same iterations. Obviously, MCSC $(\gamma=0.1, \eta=0)$ achieves more similar manifold structures as the input manifold than CSC, in comparison with Fig. 7(b), Fig. 7(c) is much more similar to Fig. 7(a). The result shown in Fig. 7(b) just gets worse reconstruction actually. In addition, we plot the manifold of MCSC with $\gamma=5$ and $\eta=0.5$ in Fig. 7(d), as expected that larger $\gamma$ and $\eta$ can improve the smoothness of the structure. Besides $\gamma$ and $\eta$, the number of samples also plays a crucial role in the structure calculation, and the results in Fig. 8 confirm that larger sample sizes result in better structures. We further select 2000 pairwise of digit ' 6 ' and evaluate the normalized correlation between the input image and its reconstruction in Fig. 9. One can see that MCSC shows a better linear fitting of $y=x$ while CSC has a bigger bias and variance.

Reconstruction Error. With respect to the reconstruction error, we calculate it in the YaleB01 dataset. In Fig. 10, the MCSC demonstrates the capability of decreasing the reconstruction loss. In addition to the manifold and reconstruction error analysis mentioned above, we also evaluate the performance on dictionary compactness and feature similarity. As theorem 1 and 2 showed that the imposing manifold constraint should have the impact on compacting dictionaries and preserving feature similarity.

Dictionary Orthogonality. To evaluate the compactness of the learned convolutional filters, we define the normal- 


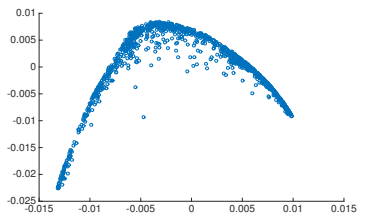

(a)

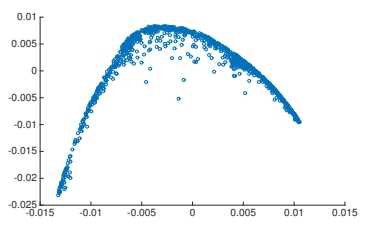

(c)

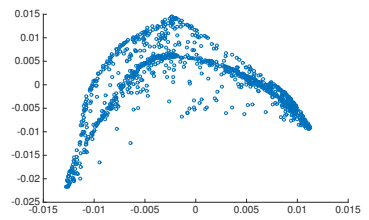

(b)

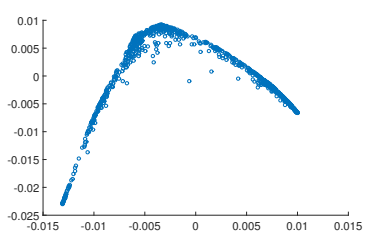

(d)
Fig. 7. Comparison of the manifold of '0' samples. (a) Manifold of the original input data; (b) Manifold of CSC; (c) Manifold of MCSC $(\gamma=$ $0.1, \eta=0)$; (d) Manifold of MCSC $(\gamma=5, \eta=0.5)$.

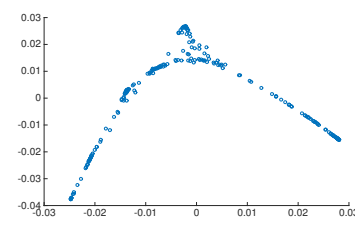

(a)

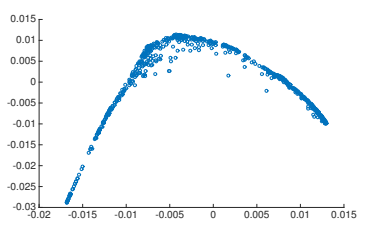

(c)

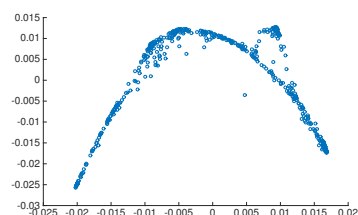

(b)

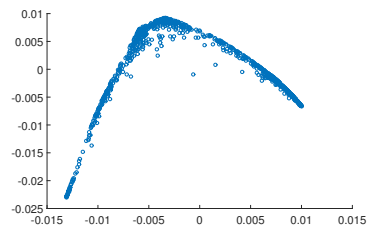

(d)
Fig. 8. Manifold of MCSC with different numbers of ' 0 ' samples. (a) 250 samples; (b) 500 samples; (c) 750 samples; (d) 1000 samples.

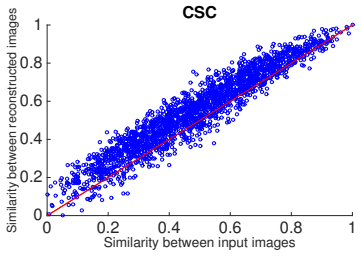

(a)

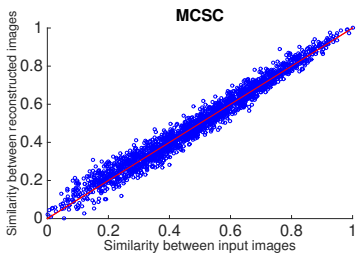

(b)
Fig. 9. (a) The similarity correspondence between the inputs and the CSC reconstructions; (b)The similarity correspondence between the inputs and the MCSC reconstructions.

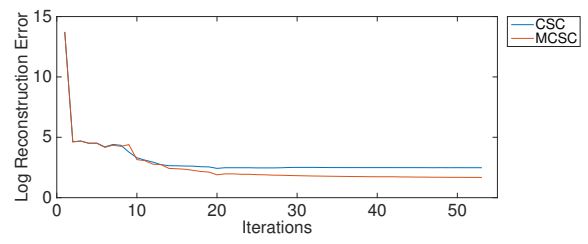

Fig. 10. Reconstruction error analysis.

ized dictionary orthogonality function for dic as

$$
\operatorname{orth}(\mathbf{d i c})=\frac{1}{K(K-1)} \sum_{i=1}^{K} \sum_{j=i+1}^{K} \operatorname{dic}_{i}^{T} \mathbf{d i c}_{j},
$$

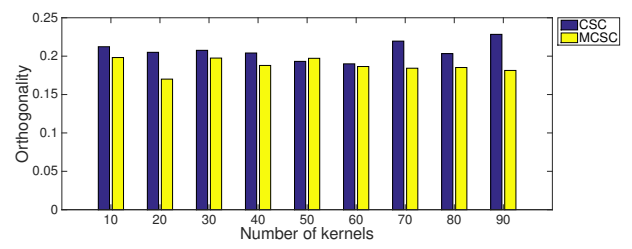

Fig. 11. Dictionary normalized orthogonal analysis with different kernel numbers of digit ' 6 '.

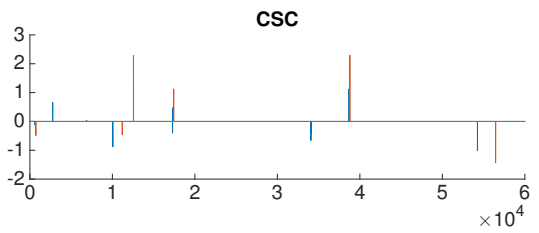

(a)

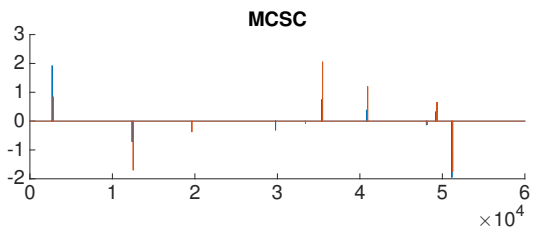

(b)

Fig. 12. (a) CSC feature maps (similarity $=0.25$ ); (b) MCSC feature maps (similarity $=0.37$ ); Noted that the similarity for the original image pair is 0.75 .

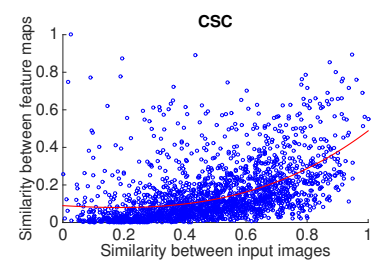

(a)

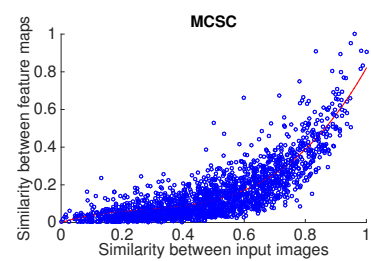

(b)
Fig. 13. (a) The similarity correspondence between the inputs and the CSC feature maps; (b) The similarity correspondence between the inputs and the MCSC feature maps.

where $\operatorname{dic}_{i}$, the $i^{\text {th }}$ column of the dic, represents a vectorized and normalized word. We calculate the normalized orthogonality in digit ' 6 ' with different kernel numbers. As shown in Fig. 11, the MCSC is more compact than that of CSC in terms of the dictionary orthogonality in most cases.

Feature Similarity. For feature similarity analysis, we plot the results of CSC and MCSC on a pair of images (the same class) sampled from YaleB data set in Fig. 12. It can be seen that the responses using the MCSC are much more similar with a higher similarity score, but the responses using the CSC vary a lot. Selecting 2000 pairwise handwritten numbers from digit ' 6 ' in USPS dataset, we calculate the relationship between their input and feature maps using the normalized correlation, and plot their similarity correspondence in Fig. 13. The MCSC similarity in Fig. 13(b) appears better fitting to $y=x$ than CSC in Fig. 13(a).

\section{Conclusions}

In this paper, we propose a novel framework of convolution sparse coding with Laplacian manifold constraint. The new 
insight of MCSC investigates mutual dependence among the observed and the reconstructed instances. Some theoretical results are obtained to explain the existing Laplacian regularization technique in sparse coding, and the reconstruction experimental results, especially in light field, show that the proposed algorithm performs favorably better against the state-of-the-art methods based on convolutional sparse coding. In future, we will investigate the new idea in this paper for tasks of feature extractions, classifications and tracking [39], [40].

\section{APPENDIX}

Lemma 1: If $\mathbf{A} \in R^{n \times n}$ and $\mathbf{B} \in R^{n \times n}$ are positive semidefinite, then

$$
\operatorname{tr}(\mathbf{A B}) \leq \operatorname{tr}(\mathbf{A}) \operatorname{tr}(\mathbf{B}) .
$$

Lemma 2: If $\mathbf{A} \in R^{n \times n}$ is positive definite and $\mathbf{B} \in R^{n \times n}$ is positive semidefinite, then

$$
\operatorname{tr}(\mathbf{A B}) \geq \frac{\operatorname{tr}(\mathbf{B})}{\operatorname{tr}\left(\mathbf{A}^{-1}\right)} .
$$

Proof of Theorem 1: In the coding update, $\mathbf{D}$ is given and $\mathbf{L}$ is positive semidefinite, we have:

$$
\operatorname{tr}\left(\mathbf{v} \mathbf{L} \mathbf{v}^{T}\right)=\operatorname{tr}\left(\mathbf{D} \mathbf{z} \mathbf{L} \mathbf{z}^{T} \mathbf{D}^{T}\right)=\operatorname{tr}\left(\mathbf{D}^{T} \mathbf{D} \mathbf{z} \mathbf{L} \mathbf{z}^{T}\right),
$$

where $\mathbf{D}^{T} \mathbf{D}$ is positive semidefinite, and $\mathbf{z L} \mathbf{z}^{T}$ positive semidefinite. We then have:

$$
\begin{aligned}
& \operatorname{tr}\left(\mathbf{v} \mathbf{L} \mathbf{v}^{T}\right)+\alpha^{\prime} \sum_{n=1}^{N} \sum_{k=1}^{K}\left\|\mathbf{z}_{k}^{n}\right\|_{2}^{2} \\
& =\operatorname{tr}\left(\mathbf{D}^{T} \mathbf{D} \mathbf{z} \mathbf{L} \mathbf{z}^{T}\right)+\alpha^{\prime} \operatorname{tr}\left(\mathbf{z}^{T} \mathbf{z}\right) \\
& \geq \operatorname{tr}\left(\mathbf{D}^{T} \mathbf{D} \mathbf{z} \mathbf{L} \mathbf{z}^{T}\right)+\frac{\alpha^{\prime}}{\operatorname{tr}(\mathbf{L})} \operatorname{tr}\left(\mathbf{z} \mathbf{L} \mathbf{z}^{T}\right) \quad \geq c_{1} \operatorname{tr}\left(\mathbf{z}^{T} \mathbf{L} \mathbf{z}\right)
\end{aligned}
$$

and

$$
\operatorname{tr}\left(\mathbf{v} \mathbf{L} \mathbf{v}^{T}\right) \leq \operatorname{tr}\left(\mathbf{D}^{T} \mathbf{D}\right) \operatorname{tr}\left(\mathbf{z} \mathbf{L} \mathbf{z}^{T}\right)=c_{2} \operatorname{tr}\left(\mathbf{z L} \mathbf{z}^{T}\right),
$$

where $c_{1}=1 / \operatorname{tr}\left(\left(\mathbf{D}^{T} \mathbf{D}+\frac{\alpha^{\prime}}{t r(\mathbf{L})} \mathbf{I}\right)^{-1}\right), c_{2}=\operatorname{tr}\left(\mathbf{D}^{T} \mathbf{D}\right)$.

Proof of Theorem 2: In the dictionary update step, $\mathbf{Z}$ is given and $\mathbf{L}$ is positive semidefinite, and we have: $\operatorname{tr}\left(\mathbf{v} \mathbf{L} \mathbf{v}^{T}\right)=\operatorname{tr}\left(\mathbf{Z} \widetilde{\mathbf{D}} \mathbf{L} \widetilde{\mathbf{D}}^{T} \mathbf{Z}^{T}\right)=\operatorname{tr}\left(\mathbf{Z}^{T} \mathbf{Z} \widetilde{\mathbf{D}} \mathbf{L} \widetilde{\mathbf{D}}^{T}\right)$, where $\widetilde{\mathbf{D}}$ is a diagonal matrix, whose diagonal entries are denoted by $\mathbf{d}$, and the zero vector has the same size of vector $\mathbf{d}$. $\|\widetilde{\mathbf{D}}\|_{F}^{2}=N\|\mathbf{d}\|_{2}^{2}$. As $\mathbf{Z}^{T} \mathbf{Z}$ and $\widetilde{\mathbf{D}} \mathbf{L} \widetilde{\mathbf{D}}^{T}$ are both positive semidefinite, we have:

$$
\begin{aligned}
& \operatorname{tr}\left(\mathbf{v} \mathbf{L} \mathbf{v}^{T}\right)+\left(\alpha^{\prime}+\beta^{\prime}\right)\|\mathbf{d}\|_{2}^{2} \\
& =\operatorname{tr}\left(\mathbf{v} \mathbf{L} v^{T}\right)+\frac{\alpha^{\prime}}{N} \operatorname{tr}\left(\widetilde{\mathbf{D}}^{\mathbf{T}} \widetilde{\mathbf{D}}\right)+\frac{\beta^{\prime}}{N}\|\widetilde{\mathbf{D}}\|_{F}^{2} \\
& \geq \operatorname{tr}\left(\mathbf{v} \mathbf{L} v^{T}\right)+\frac{\alpha^{\prime}}{N \operatorname{tr}(\mathbf{L})} \operatorname{tr}\left(\widetilde{\mathbf{D}} \mathbf{L} \widetilde{\mathbf{D}}^{T}\right)+\frac{\beta^{\prime}}{N}\|\widetilde{\mathbf{D}}\|_{F}^{2} \\
& \geq \frac{\operatorname{tr}\left(\widetilde{\mathbf{D}} \mathbf{L} \widetilde{\mathbf{D}}^{T}\right)}{\operatorname{tr}\left(\left(\mathbf{Z}^{T} \mathbf{Z}+\frac{\alpha^{\prime}}{N \operatorname{tr}(\mathbf{L})} \mathbf{I}\right)^{-1}\right)}+\frac{\beta^{\prime}}{N} \operatorname{tr}\left(\widetilde{\mathbf{D}}^{T} \widetilde{\mathbf{D}}\right) \geq c_{3}\|\mathbf{d}\|_{2}^{2},
\end{aligned}
$$

and

$$
\begin{aligned}
& \operatorname{tr}\left(\mathbf{v} \mathbf{L} \mathbf{v}^{T}\right)+\left(\alpha^{\prime}+\beta^{\prime}\right)\|\mathbf{d}\|_{2}^{2} \\
& \leq \operatorname{tr}\left(\mathbf{Z}^{T} \mathbf{Z}\right) \operatorname{tr}(\mathbf{L}) \operatorname{tr}\left(\widetilde{\mathbf{D}} \widetilde{\mathbf{D}}^{T}\right)+\left(\alpha^{\prime}+\beta^{\prime}\right)\|\mathbf{d}\|_{2}^{2}=c_{4}\|\mathbf{d}\|_{2}^{2},
\end{aligned}
$$

where $c_{3}=1 / \operatorname{tr}\left(\left(\frac{N \mathbf{L}}{\operatorname{tr}\left(\left(\mathbf{Z}^{T} \mathbf{Z}+\frac{\alpha^{\prime}}{\operatorname{Ntr}(\mathbf{L})} \mathbf{I}\right)^{-1}\right)}+\beta^{\prime} \mathbf{I}\right)^{-1}\right), c_{4}=$ $\operatorname{Ntr}\left(\mathbf{Z}^{T} \mathbf{Z}\right) \operatorname{tr}(\mathbf{L})+\alpha^{\prime}+\beta^{\prime}$.

\section{ACKNOWLEDGMENTS}

The work was supported in part by the Natural Science Foundation of China under Contract 61601466, 61672079, 61473086. The work of B. Zhang was supported in part by the Beijing Municipal Science and Technology Commission under Grant Z161100001616005. Baochang Zhang is the correspondence.

\section{REFERENCES}

[1] K. Marwah, G. Wetzstein, Y. Bando, and R. Raskar, "Compressive light field photography using overcomplete dictionaries and optimized projections," ACM Transactions on Graphics, vol. 32, no. 4, p. $46,2013.1,3,5$

[2] L. Shi, H. Hassanieh, A. Davis, D. Katabi, and F. Durand, "Light field reconstruction using sparsity in the continuous fourier domain," ACM Transactions on Graphics, vol. 34, no. 1, p. 12, 2014. 1, 3

[3] S. T. Roweis and L. K. Saul, "Nonlinear dimensionality reduction by locally linear embedding," Science, vol. 290, no. 5500, pp. 23232326, 2000. 1, 3

[4] M. Belkin and P. Niyogi, "Laplacian eigenmaps and spectral techniques for embedding and clustering." in Advances in Neural Information Processing Systems, vol. 14, 2001, pp. 585-591. 1, 3, 4, 7

[5] M. Belkin, P. Niyogi, and V. Sindhwani, "Manifold regularization: A geometric framework for learning from labeled and unlabeled examples," Journal of Machine Learning Research, vol. 7, no. Nov, pp. 2399-2434, 2006. 1, 3

[6] B. Zhang, A. Perina, V. Murino, and A. Del Bue, "Sparse representation classification with manifold constraints transfer," in Proceedings of the IEEE Conference on Computer Vision and Pattern Recognition, 2015, pp. 4557-4565. 1, 2, 5

[7] J. Lu, G. Wang, W. Deng, P. Moulin, and J. Zhou, “Multi-manifold deep metric learning for image set classification," in Proceedings of the IEEE Conference on Computer Vision and Pattern Recognition, 2015, pp. 1137-1145. 1, 2

[8] F. Heide, W. Heidrich, and G. Wetzstein, "Fast and flexible convolutional sparse coding," in Proceedings of the IEEE Conference on Computer Vision and Pattern Recognition. IEEE, 2015, pp. 51355143. 2, 4, 5, 6, 7

[9] P. Sermanet, K. Kavukcuoglu, S. Chintala, and Y. LeCun, "Pedestrian detection with unsupervised multi-stage feature learning," in Proceedings of the IEEE Conference on Computer Vision and Pattern Recognition, 2013, pp. 3626-3633. 2

[10] M. D. Zeiler, G. W. Taylor, and R. Fergus, "Adaptive deconvolutional networks for mid and high level feature learning," in Proceedings of the IEEE International Conference on Computer Vision. IEEE, 2011, pp. 2018-2025. 2

[11] Y. Zhou, H. Chang, K. Barner, P. Spellman, and B. Parvin, “Classification of histology sections via multispectral convolutional sparse coding," in Proceedings of the IEEE Conference on Computer Vision and Pattern Recognition. IEEE, 2014, pp. 3081-3088. 2

[12] M. D. Zeiler, D. Krishnan, G. W. Taylor, and R. Fergus, "Deconvolutional networks," in Proceedings of the IEEE Conference on Computer Vision and Pattern Recognition. IEEE, 2010, pp. 25282535. 2

[13] S. Gu, W. Zuo, Q. Xie, D. Meng, X. Feng, and L. Zhang, "Convolutional sparse coding for image super-resolution," in Proceedings of the IEEE International Conference on Computer Vision, 2015, pp. 1823-1831. 2

[14] Y. Zhu and S. Lucey, "Convolutional sparse coding for trajectory reconstruction," IEEE Transactions on Pattern Analysis and Machine Intelligence, vol. 37, no. 3, pp. 529-540, 2015. 2

[15] A. Cogliati, Z. Duan, and B. Wohlberg, "Piano music transcription with fast convolutional sparse coding," in Proceedings of the IEEE International Workshop on Machine Learning for Signal Processing. IEEE, 2015, pp. 1-6. 2

[16] F. D. Andilla and F. A. Hamprecht, "Sparse space-time deconvolution for calcium image analysis," in Advances in Neural Information Processing Systems, 2014, pp. 64-72. 2

[17] B. Wohlberg, "Convolutional sparse representations as an image model for impulse noise restoration," in Proceedings of the IEEE Image, Video, and Multidimensional Signal Processing Workshop. IEEE, 2016, pp. 1-5. 2 
[18] A. Serrano, F. Heide, D. Gutierrez, G. Wetzstein, and B. Masia, "Convolutional sparse coding for high dynamic range imaging," in Computer Graphics Forum. Wiley Online Library, 2016, pp. 153163. 2,7

[19] F. Heide, L. Xiao, A. Kolb, M. B. Hullin, and W. Heidrich, "Imaging in scattering media using correlation image sensors and sparse convolutional coding," Optics Express, vol. 22, no. 21, pp. 26338 26350, 2014. 2

[20] X. Luo and B. Wohlberg, "Convolutional laplacian sparse coding," in Proceedings of the IEEE Southwest Symposium on Image Analysis and Interpretation. IEEE, 2016, pp. 133-136. 2, 3

[21] S. Gao, I. W.-H. Tsang, L.-T. Chia, and P. Zhao, "Local features are not lonely-laplacian sparse coding for image classification," in Proceedings of the IEEE Conference on Computer Vision and Pattern Recognition. IEEE, 2010, pp. 3555-3561. 2, 3, 5

[22] X. Wang, R. B. Li, and J. Currey, "Leveraging $2 \mathrm{~d}$ and $3 \mathrm{~d}$ cues for fine-grained object classification," pp. 1354-1358, 2017. 2

[23] A. Serrano, E. Garces, D. Gutierrez, and B. Masia, "Convolutional sparse coding for capturing high-speed video content," Computer Graphics Forum, 2017. 2

[24] S. Boyd, N. Parikh, E. Chu, B. Peleato, and J. Eckstein, “Distributed optimization and statistical learning via the alternating direction method of multipliers," Foundations and Trends @ in Machine Learning, vol. 3, no. 1, pp. 1-122, 2011. 2, 4

[25] N. Parikh, S. P. Boyd et al., "Proximal algorithms." Foundations and Trends $($ in optimization, vol. 1, no. 3, pp. 127-239, 2014. 2, 4

[26] H. Bristow, A. Eriksson, and S. Lucey, "Fast convolutional sparse coding," in Proceedings of the IEEE Conference on Computer Vision and Pattern Recognition, 2013, pp. 391-398. 2

[27] A. Ashok and M. A. Neifeld, "Compressive light field imaging," Proceedings of SPIE, vol. 7690, no. 1, 2010. 2

[28] S. D. Babacan, R. Ansorge, M. Luessi, P. R. Mataran, R. Molina, and A. K. Katsaggelos, "Compressive light field sensing," IEEE Transactions on Image Processing, vol. 21, no. 12, pp. 4746-4757, 2012. 2

[29] Z. Xu and E. Y. Lam, "A high-resolution lightfield camera with dual-mask design," Proceedings of SPIE, 2012. 2

[30] H. Zou and T. Hastie, "Regularization and variable selection via the elastic net," Journal of the Royal Statistical Society: Series B (Statistical Methodology), vol. 67, no. 2, pp. 301-320, 2005. 3

[31] L. Yang, C. Chen, B. Zhang, and J. Han, "Adaptive multi-class correlation filters," The Pacific-Rim Conference on Multimedia, 2016. 4

[32] R. Patel and M. Toda, "Trace inequalities involving hermitian matrices," Linear Algebra and its Applications, vol. 23, pp. 13-20, 1979. 4

[33] A. S. Georghiades, P. N. Belhumeur, and D. J. Kriegman, “From few to many: Illumination cone models for face recognition under variable lighting and pose," IEEE Transactions on Pattern Analysis and Machine Intelligence, vol. 23, no. 6, pp. 643-660, 2001. 5

[34] J. J. Hull, "A database for handwritten text recognition research," IEEE Transactions on Pattern Analysis and Machine Intelligence, vol. 16, no. 5, pp. 550-554, 1994. 5, 6

[35] Y. Wu, J. Lim, and M.-H. Yang, "Object tracking benchmark," IEEE Transactions on Pattern Analysis and Machine Intelligence, vol. 37 no. 9, pp. 1834-1848, 2015. 5, 7

[36] Adams, Andrew et al. [Computer Graphics Laboratory], "Stanford light field archive," 2008, http:/ /lightfield.stanford.edu. 5

[37] J. Mairal, F. Bach, J. Ponce, and G. Sapiro, "Online learning for matrix factorization and sparse coding," Journal of Machine Learning Research, vol. 11, no. Jan, pp. 19-60, 2010. 5, 6

[38] J. Zhang, D. Zhao, and W. Gao, "Group-based sparse representation for image restoration," IEEE Transactions on Image Processing, vol. 23, no. 8, pp. 3336-3351, 2014. 5, 7

[39] B. Zhang, Z. Li, X. Cao, Q. Ye, C. Chen, L. Shen, A. Perina, and R. Ji, "Output constraint transfer for kernelized correlation filter in tracking," IEEE Transaction on Systems, Man, and Cybernetics: Systems, vol. 47, no. 4, pp. 693-703, 2017. 9

[40] B. Zhang, A. Perina, Z. Li, V. Murino, J. Liu, and R. Ji, "Bounding multiple guaasian uncertainty with application to object tracking," International Journal of Computer Vision, vol. 118, no. 3, pp. 364-379, 2016. 9
Linlin Yang received the B.S. and M.S. degrees in automation from Beihang University. His current research interests include signal and image processing, pattern recognition and computer vision.

Ce $\mathbf{L i}$ received the B.E. degree in Computer Science from Tianjin University in 2008, the M.S.and Ph.D. degrees in Computer Science from the University of Chinese Academy of Sciences in 2012 and 2015, respectively. She is currently a research assistant with China University of Mining \& Technology, Beijing, China, and supported by the Natural Science Foundation of China for Youth. Her current interests include computer vision, video analysis and machine learning.

Jungong Han is a faculty member with the School of Computing and Communications at Lancaster University, Lancaster, UK. Previously, he was a faculty member with the Department of Computer and Information Sciences at Northumbria University, UK. Dr. Han's research interests include Multimedia Content Identification, Multi-Sensor Data Fusion, Computer Vision and Multimedia Security.

Qixiang Ye received the B.S. and M.S. degrees in mechanical and electrical engineering from the Harbin Institute of Technology, Harbin, China, in 1999 and 2001, respectively, and the Ph.D. degree from the Institute of Computing Technology, Chinese Academy of Sciences, Beijing, China, in 2006. Since 2015, he has been a Full Professor with the University of Chinese Academy of Sciences, and was a Visiting Assistant Professor with the Institute of Advanced Computer Studies, University of Maryland at College Park, College Park, MD, USA, until 2013. He has published over 50 papers in refereed conferences and journals. His current research interests include image processing, visua object detection, and machine learning. Dr. Ye was a recipient of the Sony Outstanding Paper Award. He is a member of the IEEE.

Chen Chen received the B.E. degree in automation from Beijing Forestry University, Beijing, China, in 2009, the M.S. degree in electrical engineering from Mississippi State University, Starkville, MS, USA, in 2012, and the Ph.D. degree from the University of Texas at Dallas, Richardson, TX, USA, in 2016. He is currently a Postdoctoral Fellow with the Center for Research in Computer Vision, University of Central Florida, Orlando, FL, USA. His current research interests include compressed sensing, signal and image processing, pattern recognition, and computer vision. He has published over 40 papers in refereed journals and conferences in the above areas.

Xianbin Cao received the B.Eng and M.Eng degrees in computer applications and information science from Anhui University, Hefei, China, in 1990 and 1993, respectively, and the Ph.D. degree in information science from the University of Science and Technology of China, Beijing, in 1996. He is currently a Professor with the School of Electronic and Information Engineering, Beihang University, Beijing, China. He is also the Director of the Laboratory of Intelligent Transportation System. His current research interests include intelligent transportation systems, airspace transportation management, and intelligent computation.

Baochang Zhang received the B.S., M.S. and Ph.D. degrees in Computer Science from Harbin Institue of the Technology, Harbin, China, in 1999, 2001, and 2006, respectively. From 2006 to 2008, he was a research fellow with the Chinese University of Hong Kong, Hong Kong, and with Griffith University, Brisban, Australia. Currently, he is an associate professor with Beihang University, Beijing, China. His current research interests include pattern recognition, machine learning, face recognition, and wavelets.

Wanquan Liu received the B.Sc. degree in Applied Mathematics from Qufu Normal University, PR. China, in 1985, the M.Sc. degree in Control Theory and Operation Research from Chinese Academy of Science in 1988, and the Ph.D. degree in Electrical Engineering from Shanghai Jiaotong University, in 1993. He is currently an Associate Professor in the Department of Computing at Curtin University and is in editorial board for seven international journals. His current research interests include large-scale pattern recognition, siganal processing, machine learning and control systems. 